\title{
Aggregation Functionals on Complete Lattices
}

\author{
Marta Cardin ${ }^{1}$ \\ ${ }^{1}$ Department of Economics, University Ca' Foscari of Venice
}

\begin{abstract}
The aim of this paper is to introduce some classes of aggregation functionals when the evaluation scale is a complete lattice. Two different types of aggregation functionals are introduced and investigated. We consider a target-based approach that has been studied in Decision Theory and we focus on the equivalence between a utility-based approach and target-based approach. Moreover we study a class of aggregation functionals that generalizes Sugeno integrals to the setting of complete lattices.
\end{abstract}

Keywords: Completely distributive lattice, aggregation functionals, target-based aggregation, measure-based aggregation.

\section{Introduction}

Aggregation operators are an important mathematical tool for the combination of several inputs in a single outcome. that is used in pure mathematics and in many applied fields (see [7] for a general background). Real-valued fuzzy measures and their associated fuzzy integrals are widely used aggregation operators. There are many situations where inputs to be aggregated are qualitative and numerical values are used by convenience. Moreover sometimes we need to evaluate objects with a scale that is not totally ordered. As the aim of this paper is to generalize some well known aggregation functionals in a purely ordinal context. In this case only maximum and minimum are used for aggregation of different inputs.

So we study aggregation functionals based on a complete lattices and we consider in particular the class of completely distributive lattices. A general approach to aggregation on bounded posets is considered also in [3], [6] and [12].

The plan of the paper is the following. In Section 2 we introduce some background on lattices theory and we provide the necessary definitions, following by a introduction of Choquet-Stieltjes integral on real line. In Section 3 a target-based procedure to aggregate different inputs with respect to a measure is introduced developing a unifying definition for both numerical and ordinal framework. Finally we introduce a lower and upper fuzzy integrals that generalize Sugeno integral in the setting of complete lattices.

\section{Basic notions and terminology}

\subsection{General background on lattices}

A lattice is an algebraic structure $\langle L ; \wedge, \vee\rangle$ where $L$ is a nonempty set, called universe, and where $\wedge$ and $\checkmark$ are two binary operations, called meet and join, respectively, which satisfy the following axioms:

(i) (idempotency) for every $a \in L, a \vee a=a \wedge a=$ $a$

(ii) (commutativity) for every $a, b \in L, a \vee b=b \vee a$ and $a \wedge b=b \wedge a$;

(iii) (associativity) for every $a, b, c \in L, a \vee(b \vee c)=$ $(a \vee b) \vee c$ and $a \wedge(b \wedge c)=(a \wedge b) \wedge c$;

(iv) (absorption): for every $a, b \in L, a \wedge(a \vee b)=a$ and $a \vee(a \wedge b)=a$.

With no danger of ambiguity, we will denote lattices by their universes. A lattice $L$ is said to be distributive if, for every $a, b, c \in L$,

$$
\begin{aligned}
a \vee(b \wedge c) & =(a \vee b) \wedge(a \vee c) \quad \text { or, equivalently, } \\
a & \wedge(b \vee c)=(a \wedge b) \vee(a \wedge c) .
\end{aligned}
$$

As it is well-known, every lattice $L$ constitutes a partially ordered set endowed with the partial order $\leq$ given by: for every $x, y \in L$, write $x \leqslant y$ if $x \wedge y=$ $x$ or, equivalently, if $x \vee y=y$. A chain is a lattice such that for every $a, b \in L$ we have $x \leqslant y$ or $y \leqslant x$. Clearly, every chain is distributive. A lattice $L$ is said to be bounded if it has a least and a greatest element, usually denoted by 0 and 1 , respectively. A lattice $L$ is said to be complete if $\bigwedge S=\bigwedge_{x \in S} x$ and $\bigvee S=\bigvee_{x \in S} x$ exist for every $S \subseteq L$. Clearly, every complete lattice is also bounded.

For an arbitrary nonempty set $A$ and a lattice $L$, the set $L^{A}$ of all functions from $A$ to $L$ also constitutes a lattice under the operations

$$
\begin{gathered}
(f \wedge g)(x)=f(x) \wedge g(x) \quad \text { and } \\
(f \vee g)(x)=f(x) \vee g(x),
\end{gathered}
$$

for every $f, g \in L^{A}$. We denote the elements of $L$ by lower case letters $a, b, c, \ldots$, and the elements of $L^{A}$ by usual function symbols $f, g, h, \ldots$. We use $\mathbf{0}$ and $\mathbf{1}$ to denote the least element and greatest element, respectively, of $L^{A}$. Likewise and with no danger of ambiguity, for each $c \in L$, we denote by c the constant c map in $L^{A}$. If $L$ is bounded, then for each $X \subset A$, we denote by $I_{X}$ the characteristic function of $X$ in $L^{A}$, i.e.,

$$
I_{X}(x)= \begin{cases}1, & \text { if } x \in X \\ 0, & \text { otherwise }\end{cases}
$$


For further background in lattice theory we refer the reader to, e.g., Birkhoff [1], Davey and Priestley [6] and Grätzer [8].

\subsection{Completely distributive lattices}

A complete lattice $L$ is said to be completely distributive is the following distributive law holds

$$
\bigwedge_{i \in I}\left(\bigvee_{j \in J} x_{i j}\right)=\bigvee_{f \in J^{I}}\left(\bigwedge_{i \in I} x_{i f(i)}\right)
$$

for every doubly indexed subset of elements $\left\{x_{i j}\right.$ : $i \in I, j \in J\}$ of $L$.

The unit interval $[0,1]$, ordered in the natural way, is a completely distributive lattice and more generally, any complete chain is a completely distributive lattice.

In this paper we shall make use of an alternative characterization of complete distributivity introduced by Raney ([14] and [15]).

Given a complete lattice $L$ and $x, y \in L$, we write $x \triangleleft y$ and we say that $x$ is way below $y$ if and only if, whenever $X \subset L$ and $y \leq \bigvee X$ there is $z \in X$ with $x \leq z$. Then a complete lattice $L$ is completely distributive if and only if

$$
x=\bigvee\{y \in L: y \triangleleft x\} \quad \text { for each } \quad x \in L .
$$

The binary relation $\triangleleft$ satisfies the following properties:

(i) $x \triangleleft y$ implies $x \leq y$;

(ii) $z \leq x \triangleleft y \leq w$ implies $z \triangleleft w$;

(iii) $x \triangleleft y$ implies $x \triangleleft z \triangleleft y$ for some $z \in L$ (interpolation property).

As in [10] a subset $D \subset L$ is called joint-dense if $x=\bigvee\{d \in D: d \triangleleft x\}$. It can be easily proved that a a subset $D \subset L$ of a completely distributive lattice $L$ is joint-dense if and only if given $x, y \in L$ such that $x \triangleleft y$, there is $d \in L$ such that $x \triangleleft d \triangleleft y$ (see [10]). An element $x \in L$ is called supercompact (or isolated from below) if $x \triangleleft x$. A completely distributive lattice is said to be $\triangleleft$-separable if it has a countable joint-dense subset consisting of non-supercompact elements.

The lattice $[0,1]$ is an example of a $\triangleleft$-separable completely distributive lattice where we have $x \triangleleft y$ if either $x<y$ or $x=0=y$.

It can be proved that complete distributivity is a selfdual property. We can consider also the dual relation 4 and the meet-dense subsets defined as in $[2]$ and [10].

\subsection{Choquet-Stieltjes integral on $[0,1]$}

In this section we define the Choquet-Stieltjes integral as in [13] and we consider the representation of the Choquet-Stieltjes integral as a the Choquet integral.

If $A$ is anon-empty set let $(A, \mathcal{A}, m)$ be a fuzzy measure space i.e., $\mathcal{A}$ is a $\sigma$-algebra of subsets of $A$ (if $A$ is a finite set we usually assume that $A=2^{A}, m: \mathcal{A} \rightarrow[0,1]$ is a fuzzy measure such that $m(\emptyset)=0, m(A)=1$ and $m(X) \leq m(Y)$ whenever $X \subseteq Y$.

If $\overline{\mathcal{F}}$ denotes the class of [0,1]-valued measurable functions defined on $A$ and $f \in \mathcal{F}$ the Choquet integral of $f$ with respect to $m$ is defined by

$$
\int f d m=\int_{0}^{1} m_{f}(r) d r
$$

where $m_{f}(r)=m(\{x: f(x) \geq r\})$.

If $g:[0,1] \rightarrow[0,1]$ is a non-decreasing function with $g(0)=0$ and $g(1)=1$ we can define the LebesgueStieltjes measure $m_{g}$ on $[0,1]$ by

$$
m_{g}(a, b)=g(b+)-g(a-)
$$

where $g(b+)=\lim _{x \rightarrow b+} g(x)$ and $g(a-)=$ $\lim _{x \rightarrow a-g(x)}$ if $0<a, b<1$ and $g(1+)=1$, $g(0-)=0$. Now we can define the Choquet-Stieltjes integral on $[0,1]$ with respect to $m, g$ by

$$
\int f d g=\int_{0}^{1} m_{f}(r) d m_{g} .
$$

It can be proved as in [13] that if $g:[0,1] \rightarrow[0,1]$ is a continuous and increasing function with $g(0)=$ 0 and $g(1)=1$ and $f \in \mathcal{F}$ the Choquet-Stieltjes integral on $[0,1]$ with respect to $m, g$ is a Choquet integral of $g \circ f$ that is

$$
\int f d g=\int g(f) d m
$$

\section{Aggregation functionals on lattices}

\subsection{Target-based aggregation on $[0,1]^{n}$}

In this section we consider an aggregation functional on the bounded lattice $[0,1]$, defined as a functional $F: \mathcal{F} \rightarrow[0,1]$. We say that the functional $F$ is a utility-based functional if there exists a continuous and increasing function $u:[0,1] \rightarrow[0,1]$ with $u(0)=0$ and $u(1)=1$ such that

$$
F(f)=\int u(f) d m
$$

for every $f \in F$.

Moreover we say that the functional $F$ is a targetbased functional if there exists an element $b \in \mathcal{F}$ such that

$$
F(f)=m(\{r: f(r) \geq b(r)\})
$$

for every $f \in F$. It is important to note that the set $\{r: f(r) \geq b(r)\}$ belongs to $\mathcal{A}$ since the $f$ and $b$ are measurable functions.

When we consider a target-based functional we use a benchmarking procedure that ranks $[0,1]$-valued functions by the measure that they outperform the 
benchmark function $b$. A similar approach is considered in the framework of decision analysis under uncertainty where it is common to model a rational individual's preferences using utility functions. It is an alternative, mathematically equivalent, way of modeling preferences that is quite natural in many situations. We refer to the paper of Castagnoli and Li Calzi[4](and the references therein) for more details on target-based decision models.

We can easily prove that a utility-based (targetbased) functional is an aggregation functional. It is important to point out that every utility-based functional on $[0,1]$ is a target-based functional.

Proposition 1 If $u:[0,1] \rightarrow[0,1]$ is a continuous and increasing function with $u(0)=0$ and $u(1)=$ 1 there exists $a[0,1]$-valued measurable function $b$ defined on $A$ such that

$$
\int u(f) d m=m(\{x: f(x) \geq b(x)\}) .
$$

Proof We can prove that there exists a $[0,1]$ valued measurable function $b$ defined on $A$ such that $u(x)=D_{b}=m(\{r: f(r) \geq x\})$ and so the function $u$ is a cumulative distribution function of $b$. Using Choquet-Stiltjes integration we have

$$
\int u(f) d m=\int f d u=\int f d D=
$$

$\int_{0}^{1} m(\{x: f(x) \geq r\}) d D=m(\{x: f(x) \geq g(x)\})$.

\subsection{Some definitions}

Let $(A, \mathcal{A}, m)$ be a measurable space and $L$ a a bounded lattice. A fuzzy measure on $A$ with values in $L$ is a function $m: \mathcal{A} \rightarrow L$ such that $m(\emptyset)=0$, $m(A)=1$ and $m(X) \leq m(Y)$ whenever $X \subseteq Y$.

A function $f: A \rightarrow L$ is said to be measurable if the sets $\{x: f(x) \leq a\}$ and $\{x: f(x) \geq a\}$ are elements of $\mathcal{A}$ for every $a \in L$.

We denote by $\mathcal{M}$ the set of all fuzzy measures on $\mathrm{A}$ with values in $L$ and by $\mathcal{F}$ the set of the measurable functions $f: A \rightarrow L$.

A measure-based aggregation functional $F$ on $L$ is a mapping $F: \mathcal{F} \times \mathcal{M} \rightarrow L$ such that

(i) for each $m \in \mathcal{M} F(m, \mathbf{0})=0$ (boundary condition);

(ii) for each $m_{1}, m_{2}, \in \mathcal{M}$ with $m_{1} \leq m_{2}$ and $f_{1}, f_{2}, \in \mathcal{F}$ with $f_{1} \leq f_{2}$ we have $F\left(m_{1}, f_{1}\right) \leq$ $F\left(m_{2}, f_{2}\right)$ (monotonicity).

This general definition has to be completed by a variety of additional conditions depending on the considered framework.

\subsection{Target-based aggregation on completely distributive lattices}

Our aim is to extend the definition of target-based aggregation functional to an ordinal framework.
Proposition 2 If $L$ be a $\triangleleft$-separable completely distributive lattice, $m \in \mathcal{M}, b \in \mathcal{F}$ then the set $\{x: f(x) \triangleright b(x)\}$ is measurable.

Proof Let $D$ the countable join-dense subset of $L$ and $\left\{d_{n}\right\}$ an enumeration of $D$. One can easily checks that if $f, g \in \mathcal{F}$ we have that:

$$
\{x: f(x) \triangleright d\}=\bigcup_{d \triangleleft d_{n}}\left\{x: f(x) \geq d_{n}\right\} \quad \text { and }
$$

for every $d \in D$. So we can prove that for every $d \in D$ the set $\{x: f(x) \triangleright d\}$ is measurable. We have also that for every $d \in D$ the set $\{x: f(x) \triangleleft d\}$ is measurable. We can prove that $\{x: b(x) \triangleleft f(x)\}=$ $=\bigcup_{d \in D}(\{x: d \triangleleft f(x)\} \bigcap\{x: b(x) \triangleleft d\})$ and so we can conclude that the set $\{x: f(x) \triangleright b(x)\}$ belongs to $\mathcal{A}$.

Proposition 3 If If $L$ be a $\triangleleft$-separable completely distributive lattice and $b \in \mathcal{F}$ then the functional $F: \mathcal{F} \times \mathcal{M} \rightarrow L$ such that

$$
F(m, f)=m(\{x: f(x) \triangleright b(x)\})
$$

is a measure-based aggregation functional.

Proof First we note that $F$ is well-defined by Proposition 2. Then the properties of $F$ follow immediately from the properties of the relation $\triangleleft$.

If $L$ is a complete chain we have that $x \triangleleft y$ if either $x<y$ or $x=y$ is isolated from below which in this case means that $x$ is the upper endpoint of a jump in the ordering. Hence we can easily prove the following proposition.

Proposition 4 If If $L$ be a $\triangleleft$-separable complete chain and $b \in \mathcal{F}$ then the functional $F: \mathcal{F} \times \mathcal{M} \rightarrow L$ such that

$$
F(m, f)=m(\{x: f(x) \geq b(x)\})
$$

is a measure-based aggregation functional such that $F(m, \mathbf{1})=1$.

\subsection{Utility-based aggregation on complete lattices}

In this section we are interested in a class of polynomial functionals defined on a complete lattice. Following the approach in [9] we consider the functionals defined by $F_{l}, F_{u}$ :

$$
\begin{gathered}
F_{l}(m, f)=\bigvee_{x \in L}(x \wedge m(\{x: u(f(x)) \geq x\})) \text { and } \\
F_{u}(m, f)=\bigwedge_{x \in L}(x \vee m(\{x: u(f(x)) \not \leq x\})) \text { where }
\end{gathered}
$$

$u: L \rightarrow L$ is an increasing function such that $u(0)=$ 0 and $u(1)=1$. If $u$ is the identity function $F_{l}$ and $F_{u}$ extend Sugeno integral to the setting of complete lattices. 
As in [11] we introduce a generalization of the equality almost everywhere of two functions.

If $m_{1}, m_{2}, \in \mathcal{M}$ and $f_{1}, f_{2}, \in \mathcal{F}$ we say that the pairs $\left(m_{1}, f_{1}\right)$ and $\left(m_{2}, f_{2}\right)$ are equivalent, in symbols $\left(m_{1}, f_{1}\right) \sim\left(m_{2}, f_{2}\right)$ when

$$
\begin{gathered}
m_{1}\left(\left\{x: f_{1}(x) \geq a\right\}\right)=m_{2}\left(\left\{x: f_{2}(x) \geq a\right\}\right) \text { and } \\
m_{1}\left(\left\{x: f_{1}(x) \not \leq a\right\}\right)=m_{2}\left(\left\{x: f_{2}(x) \not \leq a\right\}\right)
\end{gathered}
$$

Proposition 5 Let $L$ be a complete and bounded lattice and $u: L \rightarrow L$ an increasing function such that $u(0)=0$ and $u(1)=1$.

(i) The functionals $F_{l}$ and $F_{u}$ measure-based aggregation functionals;

(ii) if $X \subseteq A$ then $F_{l}\left(m, I_{X}\right)=F_{u}\left(m, I_{X}\right)=$ $m(X)$;

(iii) if $\left(m_{1}, f_{1}\right) \sim\left(m_{2}, f_{2}\right)$ then $F\left(m_{1}, f_{1}\right)=$ $F\left(m_{2}, f_{2}\right)$.

(iv) if $L$ is completely distributive then $F_{l}(m, f)=$ $F_{u}(m, f)$ for every $m \in \mathcal{M}$ and $f \in \mathcal{F}$.

Proof Since $u$ is an increasing function and $u(0)=$ 0 and $u(1)=1$ it is easy to see that the functionals $F_{l}$ and $F_{u}$ measure-based aggregation functionals. If $X \subseteq A$ then $\left\{x: u\left(I_{X}(x)\right) \geq 1\right\}=X=\{x$ : $\left.u\left(I_{X}(x)\right) \not \leq 0\right\}$ and then $F_{l}\left(m, I_{X}\right)=F_{u}\left(m, I_{X}\right)=$ $m(X)$. By the definition of the functionals $F_{l}$ and $F_{u}$ and of the relation $\sim$ we can get (iii). Theorem 3.1 of [9] applied to the the function $u \circ f$ guarantees that $F_{l}(m, f)=F_{u}(m, f)$ for every $m \in \mathcal{M}$ and $f \in \mathcal{F}$ when $L$ is a completely distributive lattice.

\subsection{Concluding remarks}

The aim of this paper has been to consider a qualitative approach to aggregation. The focus has been on aggregation functionals defined on lattices. In particular we have introduced measure-based aggregation functionals defined on completely distributive lattices. A further research direction is that of an axiomatic characterization of the considered aggregation functionals.

\section{References}

[1] G. Birkhoff. Lattice Theory, Colloquium Pub., Vol. 25. American Mathematical Society, Providence, R.I., 1967.

[2] G. Bosi, J. Gutiérrez Garcia, and E. Indurain Unified Representability of Total Preorders and Interval Order through a Single Functions: The Lattice Approach, Order 26:255-275, Springer, 2009.

[3] M. Cardin and M. Couceiro, Invariant functionals on completely distributive lattices, Fuzzy Sets and Systems,167(1):45-56 Elsevier, 2011.

[4] E. Castagnoli and M. Li Calzi, Benchmarking real-valued acts, Games and Economics Behavior, 57:236-253, Elsevier, 2006.
[5] T. Daniëls and E. Pacuit, A General Approach to Aggregation Problems, Journal of Logic and Computation, 19(3):517-536, Oxford University Press, 2009.

[6] B.A. Davey and H.A. Priestley, Introduction to Lattices and Order, Cambridge University Press, New York, 2002.

[7] M. Grabisch, J.L. Marichal, R. Mesiar and E. Pap, Aggregation Functions, Encyclopedia of Mathematics and its Applications. Cambridge University Press, Cambridge, UK, 2009.

[8] G. Grätzer, General Lattice Theory, Birkhäuser Verlag, Berlin, 2003.

[9] G.H. Greco, Fuzzy Integral and Fuzzy Measures with Their Values in Complete Lattices, Journal of Mathemetical Analysis and Applications 126:594-603, Academic Press, 1987.

[10] J. Gutiérrez Garcia, T. Kubiak and M.A. de Prada Vicente, Insertion of lattice-valued and hedgehog-valued functions, Topology and its Application 153:1458-1475, Elsevier, 2006.

[11] E.P.. Klement, R. Mesiar, and E. Pap. A Universal Intewgral Proceedings EUSFLAT 200\%, Ostrava, vol. I:253-256, 2007.

[12] M. Komorníková and R. Mesiar, Aggregation Functions on Bounded Posets 35 years of Fuzzy sets Theory, STUDFUZZ 261:3-17, Springer, 2011.

[13] T. Murofushi and Y. Narukawa, ChoquetStieltjes Integral as a Tool for Decision Modeling, International Journal of Intelligent Systems, 23:115-127, Wiley, 2008.

[14] G. N. Raney, Completely distributive complete lattices, Proc. Amer. Math. Soc.3:677$680,1952$.

[15] G. N. Raney, A subdirect-union representation for completely distributive complete lattices, Proc. Amer. Math. Soc.4:518-522, 1953. 